\title{
Lamin A expression levels are unperturbed at the normal and mutant alleles but display partial splice site selection in Hutchinson-Gilford progeria syndrome
}

\author{
C J Reddel, A S Weiss
}

J Med Genet 2004;41:715-717. doi: 10.1136/jimg.2004.019323

$\mathrm{H}$ utchinson-Gilford progeria syndrome (HGPS) is a rare fatal genetic disorder which is characterised by the very early appearance of accelerated ageing in children. It was first described by Hutchinson ${ }^{1}$ and Gilford. ${ }^{2}$ Children with HGPS appear healthy at birth, with distinctive clinical features appearing within the first few years of life, including severe growth retardation, atherosclerosis, incomplete sexual maturation, loss of subcutaneous fat, craniofacial abnormalities, alopecia (hair loss), stiffness of joints, and other skeletal abnormalities including coxa valga (giving a "horse-riding" stance), delayed and abnormal dentition, and sclerodermalike areas of skin, with normal mental and emotional development. ${ }^{3}$

A specific causal mutation for HGPS in the LMNA gene was identified $^{45}$ and down-regulation of isoforms of the protein arising from the LMNA gene were found in HGPS patients. ${ }^{6}$ The LMNA gene encodes the A-type lamins, lamins A and C, in which several other laminopathies have their causal mutation..$^{7}$ The heterozygous de novo point mutation is located in exon 11 of the LMNA gene, which is transcribed to lamin A while the shorter lamin $\mathrm{C}$ is unaffected by the mutation. The GGC>GGT G608G single-base substitution reveals a cryptic splice site and is proposed to result in a truncated transcript encoding a protein that is 50 amino acid shorter. This mutant protein will be referred to as progerin. A less common mutation in the same codon (GGC>AGC), predicted to have similar effects on lamin A splicing, was also identified. ${ }^{4}$ Western blots reveal progerin and the normal lamin A protein in HGPS patient cells.5

This hypothesised mechanism raises questions about allelic expression and splicing efficiency. De Sandre-Giovannoli et $\mathrm{al}^{4}$ postulated that only the mutated allele may be expressed in HGPS cells. We now examine lamin A transcript levels in HGPS cells, identify their allelic sources and demonstrate that both alleles are capable of comparable levels of transcription. We further show that progerin splicing is incomplete, leading to coding transcripts derived from both alleles that encode normal lamin A.

\section{METHODS}

Skin fibroblasts were obtained from Coriell Cell Repository (NJ, USA). HGPS strains were AG03198 and AG03513B and correspond to classical forms of HGPS ( $M$ Eriksson and $F$ Collins, personal communication). Age-matched control strains were GM03348B and GM02037A. Cell culture and RNA preparation were as described. ${ }^{6}{ }^{9}$ Using DNA extracted from fibroblasts, lamin exon 11 and half of intron 11 were amplified using forward and reverse primers, with sequences GGCTCCCACTCGAGCAGCTC and GGAAGAGAAGGCAGGCT CTC, respectively. From RNA extracted from fibroblasts, lamin exons 11 and 12 were reverse-transcribed and amplified with forward and reverse primers, with sequences GGCTCCCACT GCAGCAGCTC and CATGATGCTGCAGTTCTGGG, respectively. Duplicates studies were performed in each case. For

\section{Key points}

- The major causal mutation for Hutchinson-Gilford progeria syndrome (HGPS) is in the LMNA gene where it leads to abnormal splicing of lamin A. Transcription efficiencies at the mutant and wildtype LMNA alleles were equivalent in HGPS.

- This demonstrates an absence of trans-effects and the ability of each allele to express independently.

- The mutant allele gave two types of transcripts that encoded truncated and normal lamin A.

- Abnormally spliced (progerin) transcript constituted the majority $(84.5 \%)$ of the total steady-state mRNA derived from the mutant allele.

- The abnormally spliced (progerin) transcript was a minority being $40 \%$ of all lamin A transcripts obtained from both alleles.

sequencing purposes individual products were re-amplified using the same primers. Sequencing and fragment analysis was through the Australian Genome Research Facility.

\section{RESULTS}

DNA sequencing confirms the HGPS mutation

DNA extraction, amplification, and sequencing confirmed these HGPS cell strains contained the genomic mutation ${ }^{5}$ which was missing from the control strains. RNA was extracted from HGPS and age-matched control fibroblasts and subjected to RT-PCR. Forward and reverse primers were designed to amplify exon 11 and most of the coding region of exon 12, therefore producing fragments of two sizes: one from the full length transcript (294 bp) and another fragment from the progerin transcript (144 bp). These products were obtained from HGPS strains while only the $294 \mathrm{bp}$ fragment was found for the control strains. The 294 and $144 \mathrm{bp}$ fragments of each sample were each cut and purified from agarose gels, separately re-amplified, and then sequenced. Sequence data confirmed the presence of expected lamin transcript fragments and the mutation (fig 1).

\section{Products from the mutant but not normal allele contain} a single BstXI site

The presence of the expected lamin fragments in RT-PCR products was further confirmed by fragment analysis. A 5' 6Fam primer 1 was substituted for primer in reactions and analysis by size fractionation showed that the products were within $1 \mathrm{bp}$ of their expected length (fig 2). A large amount of the 294 bp product was routinely present in all samples, with a small amount of a $208 \mathrm{bp}$ fragment that was a minor

Abbreviation: HGPS, Hutchinson-Gilford progeria syndrome 

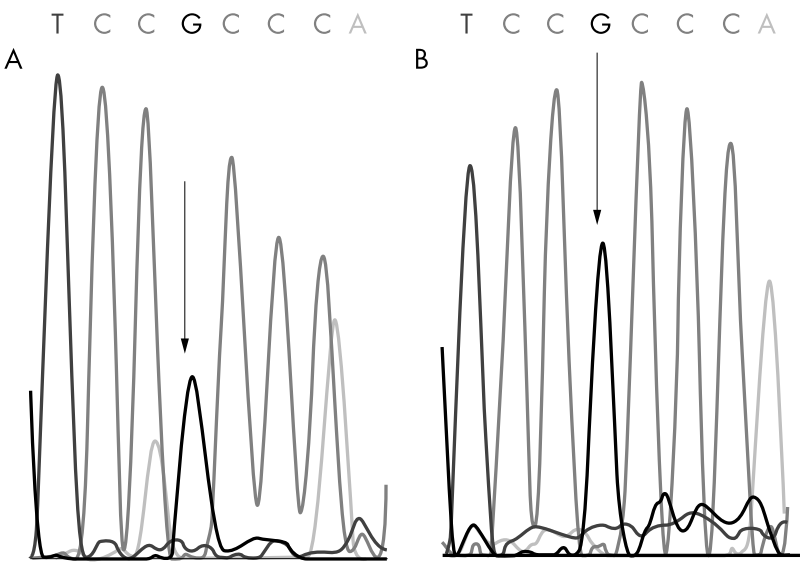

Figure 1 Critical reverse sequence of lamin CDNA of (A) HGPS line AG03198 compared to (B) age-matched control GM03348B. The site of the G608G (GGC>GGT) mutation is indicated by an arrow.

PCR artefact. The $144 \mathrm{bp}$ fragment was routinely obtained with HGPS samples. The amount of fluorescence detected for each fragment corresponds to the relative amount of fragment present and was used to determine the ratios of progerin to full-length transcripts. Progerin transcripts levels were similar ( $\sim 40 \%$ of total lamin $\mathrm{A}$ in each) in strains of HGPS cells while not present in controls. We identified a single BstXI site in LMNA exon 11 that distinguishes the wildtype and mutant forms of the full-length cDNA. Digestion of mutant $294 \mathrm{bp}$ cDNA resulted in two products of lengths 124 and $170 \mathrm{bp}$. For 5' 6-Fam-labelled RT-PCR products, only the 124 bp product was labelled and consequently detected. By definition, products with no mutation lacked the BstXI site, allowing the retention of the labelled $294 \mathrm{bp}$ fragment (fig 3). The $124 \mathrm{bp}$ fragment appeared only in digested HGPS samples and represents the correctly spliced transcript from the mutant allele. Relative fluorescence quantification defined the relative amounts of the two alleles (table 1).

\section{DISCUSSION}

Through partial sequencing of the LMNA gene, it was confirmed that one allele in HGPS skin fibroblast lines AG03198 and AG03513B contains the G608G (GGC>GGT) mutation previously observed in HGPS patients. ${ }^{45}$ Agematched controls GM03348B and GM02037A did not contain a mutant allele. This mutation was hypothesised to activate a cryptic splice site, causing a deletion of $150 \mathrm{bp}$ in lamin A mRNA. This truncated transcript codes for progerin, lamin A with a 50 aa deletion. ${ }^{45}$ The presence of the progerin mRNA transcript was confirmed in HGPS strains by amplification across the region of deletion in mRNA though the use of an RT-PCR system that generated a $294 \mathrm{bp}$ product from wildtype mRNA but a $144 \mathrm{bp}$ fragment from truncated mRNA. Analysis of RT-PCR products showed that fragments of 294 bp were amplified from HGPS and control cells, while

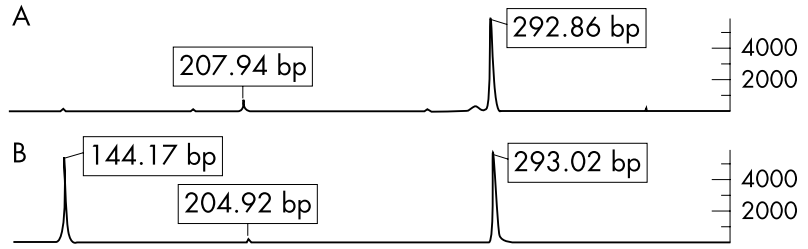

Figure 2 Representative example of plot of fragment analysis confirming the sizes of fragments from (A) control strain (GM03348B) and (B) HGPS strain (AG03198).

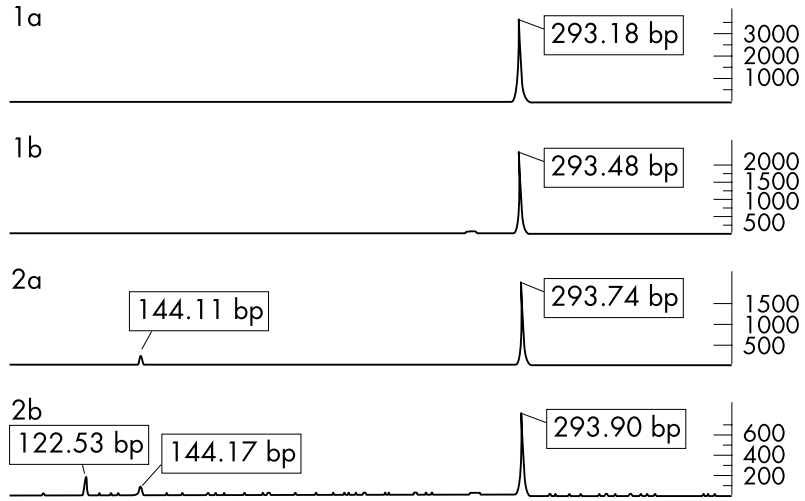

Figure 3 Representative fragment analysis of RT-PCR product digested with BstXI. (1) Control strain GM03348B, (2) HGPS strain AG03198, (a) undigested and (b) digested.

a 144 bp fragment was amplified only from HGPS cells. Separation and detection of fluorescently tagged products confirmed the expected fragment sizes and the presence of the progerin transcript in HGPS cells. Sequence data for 294 bp fragments amplified from full-length LMNA mRNA transcripts in HGPS cells revealed both the wildtype and mutant allele contributed to normally spliced product. In contrast, control cells revealed only the wildtype sequence. We conclude that transcripts arising from both alleles were present in HGPS and therefore that transcription occurred at both LMNA alleles.

These findings are important for two reasons. First, they confirm previous data suggesting that the activation of abnormal splicing in the mutant allele is incomplete. ${ }^{45} \mathrm{We}$ identified full-length transcripts from the mutant allele which encode a normal form of lamin A because the base change does not alter the glycine encoded at that site. Second, our results demonstrate that the wildtype LMNA allele is additionally expressed in HGPS. This is in contrast to the findings of De Sandre-Giovannoli et $a l^{4}{ }^{4}$ whose use of polymorphisms within the sequence led to the hypothesis that the wildtype allele of the LMNA gene may not be expressed in HGPS cells. We excluded cross-sample contamination through repeated independent and controlled amplifications, consistency of the data, the absence of a mutant peak corresponding to a mutant allele in the control cells, and consistent quantitative profiles. It is possible that the type of cell studied may have contributed to differences in results, although skin fibroblasts, used in the present study, appear to be a better choice for investigations into progeria than the lymphocytes used by De Sandre-Giovannoli and colleagues, because the disease is manifested predominantly in connective tissue. It is also possible that cells from individual

Table 1 Amounts of progerin transcripts expressed and mutant full-length cDNA, appearing as cut $124 \mathrm{bp}$ product, as percentages of the total lamin A transcript population

\begin{tabular}{|c|c|c|c|}
\hline Type & Strain & $\begin{array}{l}\text { \% Progerin } \\
\text { transcript in total } \\
\text { (SEM) }\end{array}$ & $\begin{array}{l}\text { \% Mutant full-length } \\
\text { transcript in total } \\
\text { (SEM) }\end{array}$ \\
\hline HGPS & AG03198 & $39.5 \%(2.2 \%)$ & $14.4 \%(2.5 \%)$ \\
\hline HGPS & AG03513B & $39.5 \%(2.7 \%)$ & $9.7 \%(1.4 \%)$ \\
\hline $\begin{array}{l}\text { Age-matched } \\
\text { control }\end{array}$ & GM03348B & $<1 \%$ & Undetected \\
\hline $\begin{array}{l}\text { Age-matched } \\
\text { control }\end{array}$ & GM02037A & Undetected & Undetected \\
\hline
\end{tabular}

The term "mutant full-length transcript" refers to the correctly spliced transcript from the mutant allele. SEM, standard error of the mean. 
DNA - LMNA gene
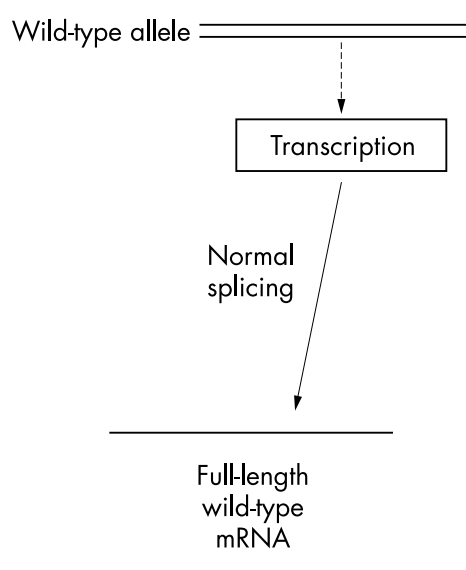

$53 \%$

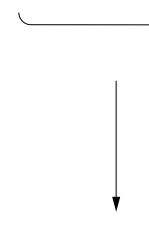

Normal

$\operatorname{lamin} \mathrm{A}$

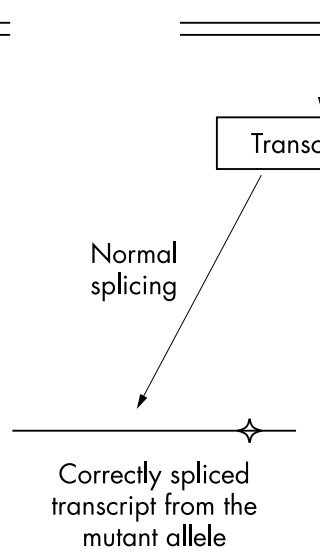

$7 \%$

$60 \%$
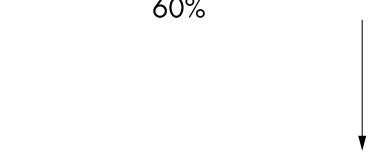

Normal

$\operatorname{lamin} \mathrm{A}$
Figure 4 Quantitative schematic showing new hypothesised model of allelic expression and abnormal splicing event in HGPS cells. A star indicates the location of the $G 608 \mathrm{G}$ (GGC>GGT) mutation.
HGPS patients may vary in their responses (which may also affect severity of the disease in vivo) but this does not diminish the conclusion that HGPS fibroblasts are capable of comparable levels of transcription where each allele is expressed and the mutant allele undergoes both normal and abnormal splicing.

Quantitative analysis of labelled full-length and progerin products revealed that the progerin transcript constituted $40 \%$ of the total lamin A transcripts. These findings were similar in the HGPS strains. In contrast, each control contained undetectable levels of progerin transcript. BstXI digestion enabled a distinction between full-length wildtype transcripts and the correctly spliced transcripts from the mutant allele, which were originally identical in size and differed by a single nucleotide. From these studies, the correctly spliced transcript from the mutant allele contributed $12-14 \%$ of the total full-length transcript. Considering that the full-length transcript was $60 \%$ of the total, this means that wildtype full-length transcript constituted around 53\% of all lamin A transcript while correctly spliced transcript from the mutant allele contributed $7 \%$ of the total transcript population. Approximately half of the total lamin A transcripts arose from each allele, and $84.5 \%$ of transcript arising from the mutant allele was abnormally spliced $(39.5 \%$ of the total). Each allele contributed approximately half of the steady-state transcript level (fig 4).

Several as yet uncharacterised factors should be the subject of further study. First, the present study was not able to make a direct comparison between whole transcription levels of lamin A in different cell lines. Second, the relative quantities and stability of translated progerin have not yet been established.

These findings raise the question of how a minor level of transcript and presumably minor amounts of mutant protein can cause the disease phenotype in the presence of regular lamin A. Progerin presumably functions as a dominant negative by interfering with the structure of the nuclear lamina, intranuclear architecture, and macromolecular interactions that collectively would have a major impact on nuclear function.

\section{ACKNOWLEDGEMENTS}

We thank the Progeria Research Foundation for their support, the families with HGPS who donated skin samples, and the Progeria Research Consortium.

\section{Authors' affiliations}

C J Reddel, A S Weiss, School of Molecular and Microbial Biosciences, University of Sydney, NSW 2006, Australia

Conflict of interest: none declared.

Correspondence to: Professor Anthony Weiss, School of Molecular and Microbial Biosciences, University of Sydney, NSW 2006, Australia; a.weiss@mmb.usyd.edu.au

Revised version received 25 March 2004

Accepted for publication 29 March 2004

\section{REFERENCES}

1 Hutchinson J. A case of congenital absence of hair with atrophic condition of the skin and its appendages. Lancet 1886;1:923.

2 Gilford H. Progeria: a form of senilism. Practitioner 1904;73:188-217.

3 DeBusk FL. The Hutchinson-Gilford progeria syndrome. Report of 4 cases and review of the literature. J Pediatr 1972;80:697-724.

4 De Sandre-Giovannoli A, Bernard R, Cau P, Navarro C, Amiel J, Boccaccio I, Lyonnet S, Stewart CL, Munnich A, Le Merrer M, Levy N. Lamin A truncation in Hutchinson-Gilford progeria. Science 2003;300:2055.

5 Eriksson M, Brown WT, Gordon LB, Glynn MW, Singer J, Scott L, Erdos MR, Robbins CM, Moses TY, Berglund P, Dutra A, Pak E, Durkin S, Csoka AB, Boehnke M, Glover TW, Collins FS. Recurrent de novo point mutations in lamin A cause Hutchinson-Gilford progeria syndrome. Nature 2003;423:293-8.

6 Robinson L, Karlsson NG, Weiss AS, Packer NH. Proteomic analysis of the genetic premature aging disease Hutchinson Gilford progeria syndrome reveals differential protein expression and glycosylation. J Proteome Res 2003:2:556-7.

7 Burke B, Stewart CL. Life at the edge: the nuclear envelope and human disease. Nat Rev Mol Cell Biol 2002;3:575-85.

8 Novelli G, D'Apice MR. The strange case of the "lumper" lamin A/C gene and human premature ageing. Trends Mol Med 2003:9:370-5.

9 O'Brien ME, Weiss AS. Hutchinson-Gilford progeria fibroblasts exhibit metabolically normal uridine uptake and RNA synthetic rates. Biochem Biophys Res Commun 1995;210:225-30. 